A.M. Steinberg, C.M. Arndt, W. Meier, Parametric study of vortex structures and their dynamics in swirl-stabilized combustion, Proc. Combust. Inst. 34 (2013) 3117-3125

The original publication is available at www.elsevier.com

http://dx.doi.org/10.1016/j.proci.2012.05.015 


\title{
Parametric study of vortex structures and their dynamics in
}

\section{swirl-stabilized combustion}

\author{
A. M. Steinberg ${ }^{a}$, C. M. Arndt ${ }^{b}$, and W. Meier ${ }^{b}$ \\ ${ }^{a}$ Institute for Aerospace Studies, University of Toronto, Toronto M3H 5T6, Canada \\ ${ }^{b}$ Institut für Verbrennungstechnik, Deutsches Zentrum für Luft- und Raumfahrt (DLR), 70569 Stuttgart, Germany
}

\section{Abstract}

The dynamics of major flow structures were studied in a gas turbine model combustor for perfectly premixed swirlstabilized flames under a variety of reacting and non-reacting conditions using high-repetition-rate laser diagnostics. The studied combustor is a target case for the International Workshop on Advanced Measurement Techniques and Computational Methods for Premixed and Partially Premixed Combustion. Measurements were taken of the three-component velocity field, $\mathrm{OH}$ planar laser induced fluorescence, and $\mathrm{OH}^{*}$ chemiluminescence at a rate of $10 \mathrm{kHz}$ for nine different flow conditions, covering a range of thermal powers $\left(P_{t h}=10-35 \mathrm{~kW}\right)$ and equivalence ratios $(\phi=0.65-0.8)$. Under all non-reacting conditions, the dominant flow structure was a helical vortex core (HVC) that rotated around the burner at a frequency represented by a constant Strouhal number $\mathrm{St}_{H, N R}=0.78$. However, igniting the burner significantly altered the flow structures. At most conditions, the strength and frequency of the HVC increased relative to the corresponding non-reacting case. The HVC frequency in such cases was once again represented by a constant Strouhal number of $\mathrm{St}_{H, R}=0.88$, irrespective of the thermal power or equivalence ratio. The HVC frequency was considerably higher than the frequency of the self-excited thermo-acoustic oscillations exhibited by the burner. However, at other conditions, combustion prevented formation of the HVC. In such cases, the dominant flow structure dynamics were periodic shear layer oscillations and shedding of toroidal vortices at the thermo-acoustic frequency. Cases in which combustion prevented formation of the HVC included those at low thermal powers $\left(P_{t h} \leq 15 \mathrm{~kW}\right)$ and the highest equivalence ratio $(\phi=0.8)$. A distinct relationship was found between the flow structure geometry and the pressure oscillation amplitude, with cases having an HVC resulting in higher pressure oscillations.

Keywords: Thermo-acoustic instabilities, swirl flows, high-repetition-rate laser diagnostics, helical vortices, gas turbine combustion

\section{Introduction}

This paper reports a systematic investigation of flow structure dynamics in a swirl-stabilized combustor, conducted using measurements from high-repetition-rate laser diagnostics. Such swirl-stabilized combustors are often employed in modern gas turbine engines, as they provide an effective means of stabilizing the fuel-lean premixed flames that are used to reduce $\mathrm{NO}_{\mathrm{x}}$ 
emissions [1-3]. However, lean premixed combustion systems have a tendency to self-excite large amplitude pressure and heat release oscillations, or thermo-acoustic instabilities. These oscillations lead to corresponding oscillations in the mechanical and thermal loads on various engine components, which can cause lifetime reduction and/or premature failure. Thermo-acoustic instabilities also can cause reduced combustion efficiency, increased pollutant emissions, flame blow-off, and flame flash-back $[2,3]$.

Unfortunately, the physical phenomena that couple heat release and pressure oscillations to excite thermo-acoustic instabilities are very complex. One of the most important mechanisms is flow-field coupling, wherein thermo-acoustically induced velocity oscillations lead to periodic changes in the heat release rate [4-9]. In swirl-stabilized combustors particularly, very complex flow structures can form and interact with the flame [10-19]. For example, swirl induced vortex breakdown leads to the formation of a large central recirculation zone (CRZ) downstream of the nozzle exit, which transports burned gas to the flame base and stabilizes the combustion $[10-12,17]$. The swirling flow also may lead to the formation of unsteady, large-scale, three-dimensional vortex structures, such as helical vortex cores [10-19]. These dynamic flow structures spiral around the burner axis and rotate around the nozzle, affecting the mixing and combustion processes. Steinberg et al. [8, 9] recently showed that periodic 3D flow-flame interactions can be a dominant pathway for thermo-acoustic energy transfer in swirl-stabilized combustion.

Hence, understanding the dynamic behavior of flow structures in swirl-stabilized combustors is important for better predicting and controlling thermo-acoustic instabilities. Of particular interest is the behavior of the three-dimensional helical vortex modes. Previous experimental and numerical studies have characterized several aspects of these flow structures, and have shown that combustion can greatly alter their behavior [10-19]. In some situations, combustion can inhibit the formation of structures that are present in a corresponding non-reacting flow, while in other situations, combustion can increase the strength of the structures $[13,16]$. Moreover, heat release affects the frequency and shape of the structures in a complex manner that can depend on the thermal power, equivalence ratio, geometry, swirl number, method of fuel injection, and other parameters [16].

This work investigates the influence of heat release, thermal power, and equivalence ratio on the existence and dynamics of flow structures in a gas turbine model combustor operating with perfectly premixed methane-air flames. The combustor is a derivative of an industrial design by Turbomeca S. A., which has previously been studied both experimentally [20-23] and computationally [13, 24-27]. Because of the relatively straightforward geometry and rich wealth of phenomena exhibited by this burner, it was selected as a target case by the International Workshop on Advanced Measurement Techniques and Computational Methods for Premixed and Partially Premixed Combustion. However, the previous experimental work in this burner was performed in a 'technically' premixed combustion mode, wherein fuel was injected into the swirler and did not perfectly premix before reaching the flame. Pressure oscillations in this technically premixed configuration lead to periodic changes in the equivalence ratio at the flame, which was identified as a major thermo-acoustic coupling mechanism. Conversely, simulations of this burner (with the exception of recent work by Franzelli et al. [27]) typically have considered the flow to be perfectly premixed.

The present study offers a new, comprehensive data set on this burner. A total of nine perfectly premixed conditions were studied, covering a range of thermal powers and equivalence ratios. Several 'technically premixed' cases also were measured, but are not reported here. Data from $10 \mathrm{kHz}$ repetition-rate laser and optical diagnostics were collected for both non-reacting 


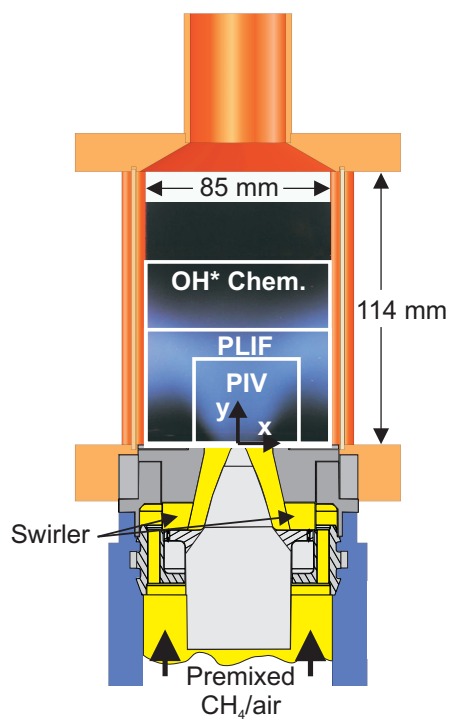

Fig. 1: Combustor schematic with the diagnostic fields-of-view indicated.

\begin{tabular}{lcccc}
\hline \hline Case & $\phi$ & $\begin{array}{c}P_{t h} \\
(\mathrm{~kW})\end{array}$ & $\begin{array}{c}\dot{m}_{\text {air }} \\
(\mathrm{g} / \mathrm{min})\end{array}$ & $\begin{array}{c}\dot{m}_{C H 4} \\
(\mathrm{~g} / \mathrm{min})\end{array}$ \\
\hline 1R \& NR & 0.70 & 10 & 295 & 12.0 \\
2R \& NR & 0.70 & 15 & 440 & 18.0 \\
3R \& NR & 0.70 & 20 & 588 & 24.0 \\
4R \& NR & 0.70 & 25 & 733 & 30.0 \\
\hline 5iR \& NR & 0.65 & & 940 & 36.0 \\
5R \& NR & 0.70 & & 880 & 36.0 \\
5iiR \& NR & 0.75 & 30 & 824 & 36.0 \\
5iiiR \& NR & 0.80 & & 770 & 36.0 \\
\hline 6R \& NR & 0.70 & 35 & $1.02 \times 10^{3}$ & 42.0 \\
\hline
\end{tabular}

Table 1: Test cases. $R$ and $N R$ represent reacting and non-reacting conditions, respectively.

and reacting flows at each condition. These data are used to describe the influence of the various parameters on the major flow structures.

\section{Experiment and Diagnostics}

\subsection{Combustor and test conditions}

The gas turbine model combustor is shown in Fig. 1, and the test conditions are given in Table 1. The experiment covered nine flow conditions, involving two parameter variations. In the first, the equivalence ratio was held constant at $\phi=0.7$ and the thermal power was varied from $P_{t h}=10-35 \mathrm{~kW}$ in $5 \mathrm{~kW}$ increments (Cases 1-6). In the second, the $P_{t h}$ was held constant at $30 \mathrm{~kW}$ and the equivalence ratio was varied between $\phi=0.65-0.80$ in 0.05 increments (Cases 5i-5iii, including Case 5 at $\phi=0.7)$. For each condition, measurements were taken both for reacting (Cases XR) and non-reacting (Cases XNR) flows.

All flow rates were controlled using electromechanical mass flow controllers (Brooks) and monitored using calibration standard Coriolis mass flow meters (Siemens Sitrans F C) with an uncertainty of 1.5\%. The methane fuel was perfectly premixed with dry air using a commercial premixer approximately $2 \mathrm{~m}$ upstream of the combustor plenum. This fuel/air mixture then passed through a choked orifice plate located $0.15 \mathrm{~m}$ upstream of the plenum. The orifice diameter was adjusted and the upstream pressure monitored to ensure choked flow at all conditions. The fuel/air mixture was then fed via the plenum $(\varnothing=78 \mathrm{~mm})$, through the radial swirler $(12$ swirl vanes $)$, to the burner nozzle $(\varnothing=27.85 \mathrm{~mm}$ exit $)$, and then into the 
combustion-chamber $(85 \times 85 \mathrm{~mm}$ cross-section, $114 \mathrm{~mm}$ length). The inner surface of the nozzle was formed by a conical bluff body, the tip of which was at the nozzle exit plane (nozzle inner diameter $=0 \mathrm{~mm}$ at $y=0 \mathrm{~mm}$ ). The combustion-chamber itself was composed of four large fused-silica windows, held in the corners by Inconel posts, and topped with a conical steel plate and exhaust tube $(\varnothing=40 \mathrm{~mm})$. The large windows provided good optical access to the combustion-chamber, allowing for the application of optical diagnostics. During a data acquisition run, approximately $5 \%$ of the air mass flow was diverted through a fluidized bed particle seeder containing $\mathrm{TiO}_{2}$ particles with a diameter of approximately $1 \mu \mathrm{m}$ to enable the velocity measurements described below. The mean flow field measured in this burner contained the large central recirculation zone and smaller corner recirculation zones that are characteristic of confined swirl-stabilized combustors [21].

The system was equipped with multiple ports for pressure and temperature measurements. Pressures were measured using calibrated microphone probes (Brüel \& Kjaer, Type 4939), sampled at a rate of $50 \mathrm{kHz}$. During these measurements, the combustion-chamber pressure was measured using two probes in a corner post, and the plenum pressure was measured using a probe flush with the plenum wall. However, detailed acoustic characterization using multiple ports and Comsol simulations (not described here) have shown that the system operates as a multi-chamber Helmholtz resonator. Hence, the thermo-acoustic pressure oscillation was essentially spatially uniform throughout each of the combustion and plenum chambers, with significant spatial pressure gradients only within the swirler/nozzle assembly and exhaust tube.

Data on the burner temperature, along with run-to-run atmospheric conditions are available from the authors for simulation development. Furthermore, composition and temperature data inside the combustion-chamber have been measured using Raman scattering for select conditions, and are available for simulation evaluation.

\subsection{Diagnostics}

For each reacting test case, the planar three-component velocity field was measured using stereoscopic particle image velocimetry (S-PIV), the OH radical distribution was measured using planar laser induced fluorescence (PLIF), and the lineof-sight integrated chemiluminescence from electronically excited $\mathrm{OH}\left(\mathrm{OH}^{*}\right)$ was measured using direct light imaging; only S-PIV was performed for non-reacting cases. All measurements were conducted at a sustained repetition-rate of $10 \mathrm{kHz}$. A minimum of two independent data acquisitions were conducted at each condition, resulting in over 15000 measurements from each camera at each condition.

The S-PIV system consisted of a high-repetition-rate, dual-cavity, diode-pumped solid-state (DPSS) Nd:YAG laser (Edgewave, IS-6IIDE) and a pair of high-speed CMOS cameras (LaVision HSS 8). Laser pulse pairs (532 nm, $2.6 \mathrm{~mJ} / \mathrm{pulse}, 14 \mathrm{~ns}$ pulse duration, $5-15 \mu$ s between pulses depending on conditions) repeating at $10 \mathrm{kHz}$ were expanded into a sheet having a useable height of about $40 \mathrm{~mm}$ and a full-width-at-half-maximum (FWHM) of approximately $1 \mathrm{~mm}$ at the measurement area. Particle-scattered light from the $\mathrm{TiO}_{2}$ seed was collected into the cameras using $100 \mathrm{~mm}$ focal length commercial camera lenses (Tokina) set to $f / 5.6$. The field-of-view imaged by the PIV system was approximately $49 \mathrm{~mm} \times 38 \mathrm{~mm}$. Due to optical access restrictions caused by the combustion chamber posts, the S-PIV field-of-view was offset from the centerline of the burner, covering the range $x \approx-17$ to $32 \mathrm{~mm}$.

The CMOS cameras were mounted equidistantly from opposite sides of the laser sheet in an angular forward-scatter configuration. Standard corrections were made for image defocusing and perspective distortion using Scheimpflug adapters and a 3D dot target (LaVision Type 7). The same target images were used to align the fields-of-view from the PIV cameras with those from the PLIF and chemiluminescence cameras described below. Vector fields were computed from the particle 


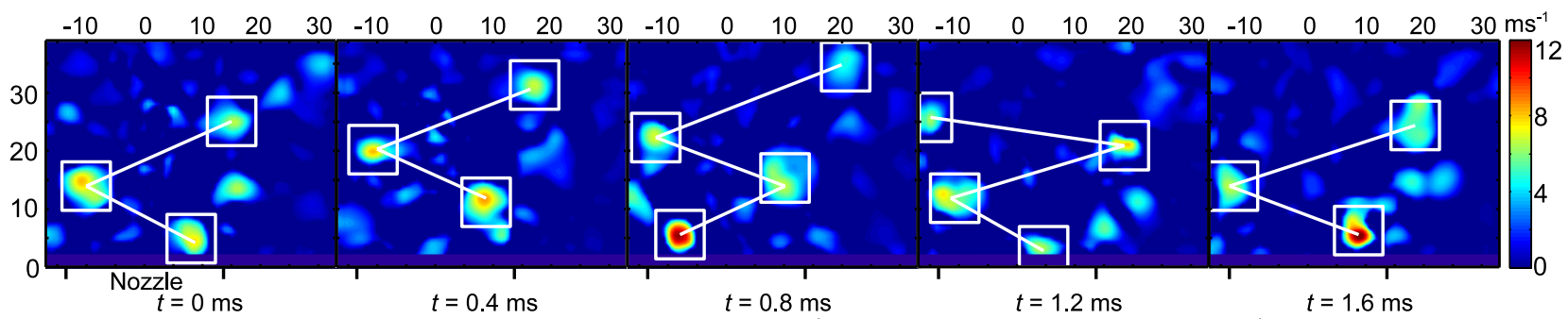

Fig. 2: Typical dynamics of the swirling strength field for Case $5 \mathrm{NR}\left(P_{t h}=30 \mathrm{~kW}, \phi=0.7\right.$, non-reacting). The measurements show a helical vortex structure that rotates around the burner in about $1.6 \mathrm{~ms}$. Axis units are millimeters.

image spatial cross-correlation using the LaVision DaVis 8.0 software package, with a spatial resolution and vector spacing of approximately $1 \mathrm{~mm}$ and $0.5 \mathrm{~mm}$ respectively.

The OH PLIF system consisted of a DPSS laser (Edgewave IS8II-E) pumping a dye laser (Sirah Credo). The dye laser output was frequency doubled and tuned to excite the $\mathrm{Q}_{1}(7)$ line of the $\mathrm{A}-\mathrm{X}\left(v^{\prime}=1, v^{\prime \prime}=0\right)$ band of $\mathrm{OH}$ at $283.2 \mathrm{~nm}$, with a final laser energy of approximately $0.18 \mathrm{~mJ} /$ pulse. The laser beam was expanded into a sheet with a useable height of approximately $50 \mathrm{~mm}$ and a FWHM of approximately $0.4 \mathrm{~mm}$ at the measurement area, combined with the sheet from the PIV laser using a dichroic mirror, and transmitted through the combustor along the same beam path. The PLIF laser pulse occurred between PIV laser pulses for each velocity field.

Fluorescence of the $\mathrm{OH}$ radical in the range of $310 \mathrm{~nm}$ was acquired with a CMOS camera (LaVision HSS5) equipped with an external, two-stage, lens-coupled intensifier (LaVision HS-IRO) and a $45 \mathrm{~mm} \mathrm{f/1.8} \mathrm{UV} \mathrm{lens} \mathrm{(Cerco).} \mathrm{The} \mathrm{field-of-view}$ of the PLIF system covered the entire width of the combustion chamber $(85 \mathrm{~mm})$ and extended from the nozzle exit to a height of $50 \mathrm{~mm}$. Background luminosity and elastic scattering were reduced by using a short intensifier gate (100 ns) and a high-transmission (> $>0 \%$ at $310 \mathrm{~nm}$ ) bandpass interference filter (Laser Components $\mathrm{GmbH}$ ). The OH images were corrected for the mean laser sheet intensity profile, which was determined based on 1000 images of the fluorescence from a uniform acetone vapor that was doped into the test area. The PLIF images were filtered with a 0.4 mm Gaussian filter (same width as the sheet thickness) prior to further analysis to reduce high-frequency pixel noise. This sheet thickness and filter width set the effective spatial resolution of the PLIF images.

The line-of-sight integrated chemiluminescence emission from $\mathrm{OH}^{*}$ was imaged using an identical camera/lens/filter arrangement to that in the OH-PLIF system. However, the intensifier gate time was extended to $15-25 \mu$ s (depending on conditions) in order to capture sufficient signal. The $\mathrm{OH}^{*}$ signal can be taken as a qualitative indicator of the integrated heat release rate in the combustor $[28]$.

\section{Results and analysis}

To begin, temporal sequences of typical flow field and flame dynamics are presented that demonstrate important phenomena. This is followed by an analysis of the frequencies and strengths of the flow and pressure oscillations. The geometry and dynamics of the major flow structures varied considerably over the studied parameter set. In the non-reacting flows, a helix shaped vortical mode was present at all conditions. This helical vortex core (HVC) was the most energetic dynamic flow structure at all non-reacting conditions. Similarly, at most high thermal power reacting cases $\left(P_{t h}>15 \mathrm{~kW}, \phi<0.8\right)$, the helical mode was present and was stronger (higher vorticity and swirling strength) than in the corresponding non-reacting cases. However, combustion prevented formation of the HVC for low thermal power and high equivalence ratio reacting cases

$\left(P_{t h} \leq 15 \mathrm{~kW}\right.$ or $\left.\phi=0.8\right)$. The dominant dynamic flow structures in these cases were symmetric (toroidal) vortices that were 


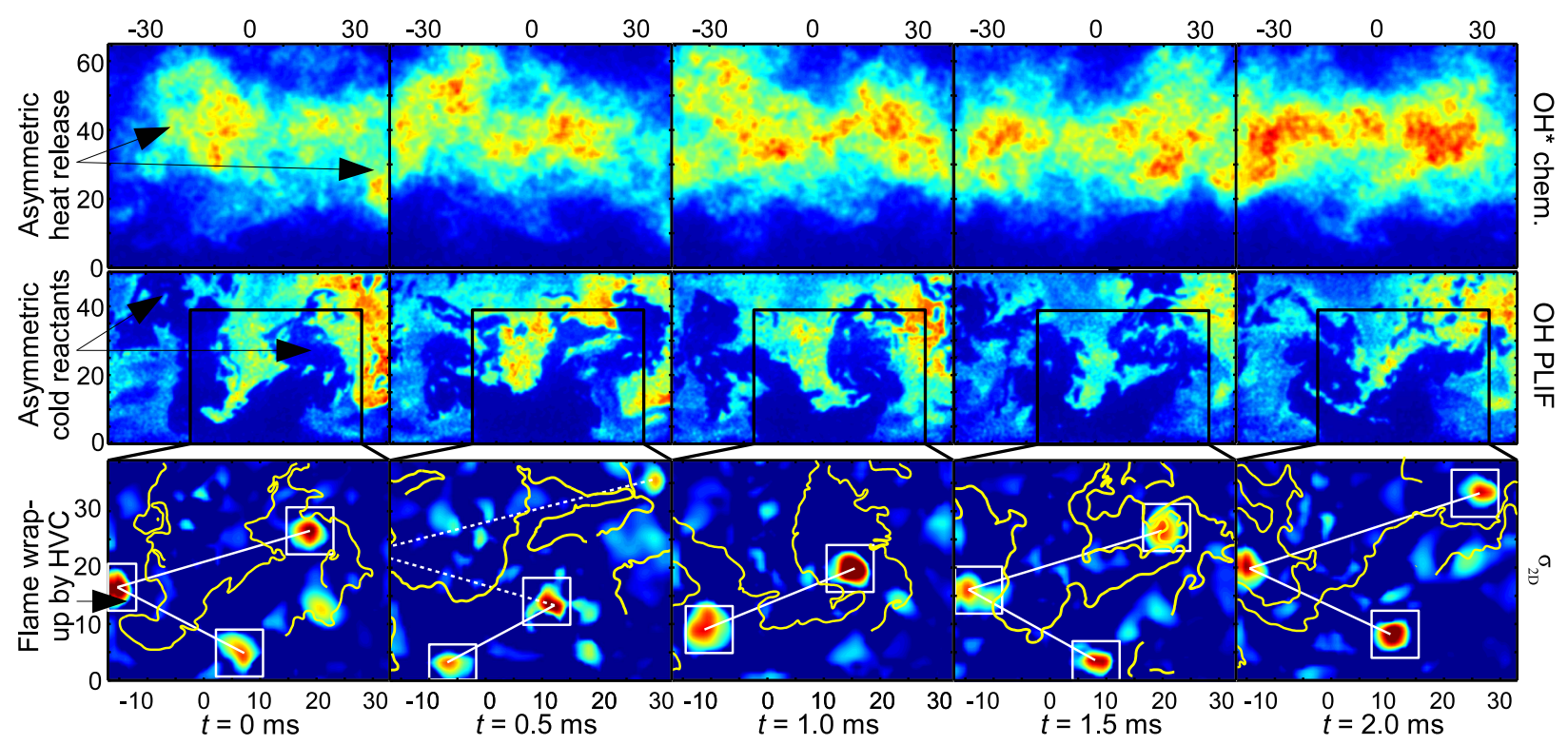

Fig. 3: Typical dynamics of the $\mathrm{OH}^{*}$ chemiluminescence, $\mathrm{OH} \mathrm{PLIF}$, and swirling strength fields for Case $5 \mathrm{R}\left(P_{t h}=30 \mathrm{~kW}, \phi=0.7\right.$, reacting). The measurements show dynamic heat release asymmetries, reactant flow asymmetries, and flame corrugation asymmetries associated with a helical vortex. The swirling strength color scale is the same as in Fig. 2. The color scale for the flame images is between 0 and a fixed count level across all images. Axis units are millimeters.

shed from the shear layers between in inflow and the central recirculation zone (CRZ).

\subsection{Temporal flow and flame dynamics}

The basic features described above are shown in Figs. 2 - 4. The temporal sequences are taken from Case $1 \mathrm{R}\left(P_{t h}=10 \mathrm{~kW}\right.$, $\phi=0.7)$ and Cases $5 \mathrm{R} \& \mathrm{NR}\left(P_{t h}=30 \mathrm{~kW}, \phi=0.7\right)$. In these figures, the combustion is shown by the line-of-sight integrated $\mathrm{OH}^{*}$ chemiluminescence field (qualitative heat release) and/or the OH PLIF field (planar hot product distribution). The flow field is visualized by the $2 \mathrm{D}$ swirling strength $\left(\sigma_{2 D}\right)$. The swirling strength is defined as the magnitude of the imaginary part of velocity gradient tensor eigenvalues, and represents the rate at which local streamlines spiral around a center point in a reference frame moving with the flow [29]. From planar measurements, the 2D swirling strength represents the rate at which local 2D streamlines rotate around a point in the plane. It is noted that the exact definition of $\sigma_{2 D}$ in terms of swirling rate is strictly valid only where the flow is locally isodensity, which may not be the case in the present reacting flows. However, as will be seen below, $\sigma_{2 D}$ provides a good visualization of the flow structures.

Figure 2 shows images from a temporal sequence of $\sigma_{2 D}$ fields from Case 5NR. Coherent vortices were present in an expanding zig-zag pattern that crossed the burner nozzle. This is the planar signature of a coherent HVC that spirals around the burner axis. The HVC rotates around the combustor at a frequency that is dictated by the precession of the vortex base. Hence, the helix is sometimes referred to as a precessing vortex core (PVC). The period of the vortex motion around the combustor in this case was about $1.6 \mathrm{~ms}$.

Figure 3 shows a temporal sequence from the corresponding reacting conditions, Case $5 R$. In this figure, the swirling strength fields are superimposed by curves representing the topography of the high OH-PLIF gradients, which roughly represent the flame topography. Similar to the non-reacting case, the reacting case contains an HVC. In general, $\sigma_{2 D}$ in the HVC was greater than in the non-reacting case. Considerable flow and flame asymmetries occurred throughout the time sequence. At $t=0 \mathrm{~ms}$, the majority of the heat release was on the right side of the image. There was a distinct asymmetry in the cold inflowing reactants (low $\mathrm{OH}$ signal), with reactants on the left side penetrating much farther into the combustor. This was 

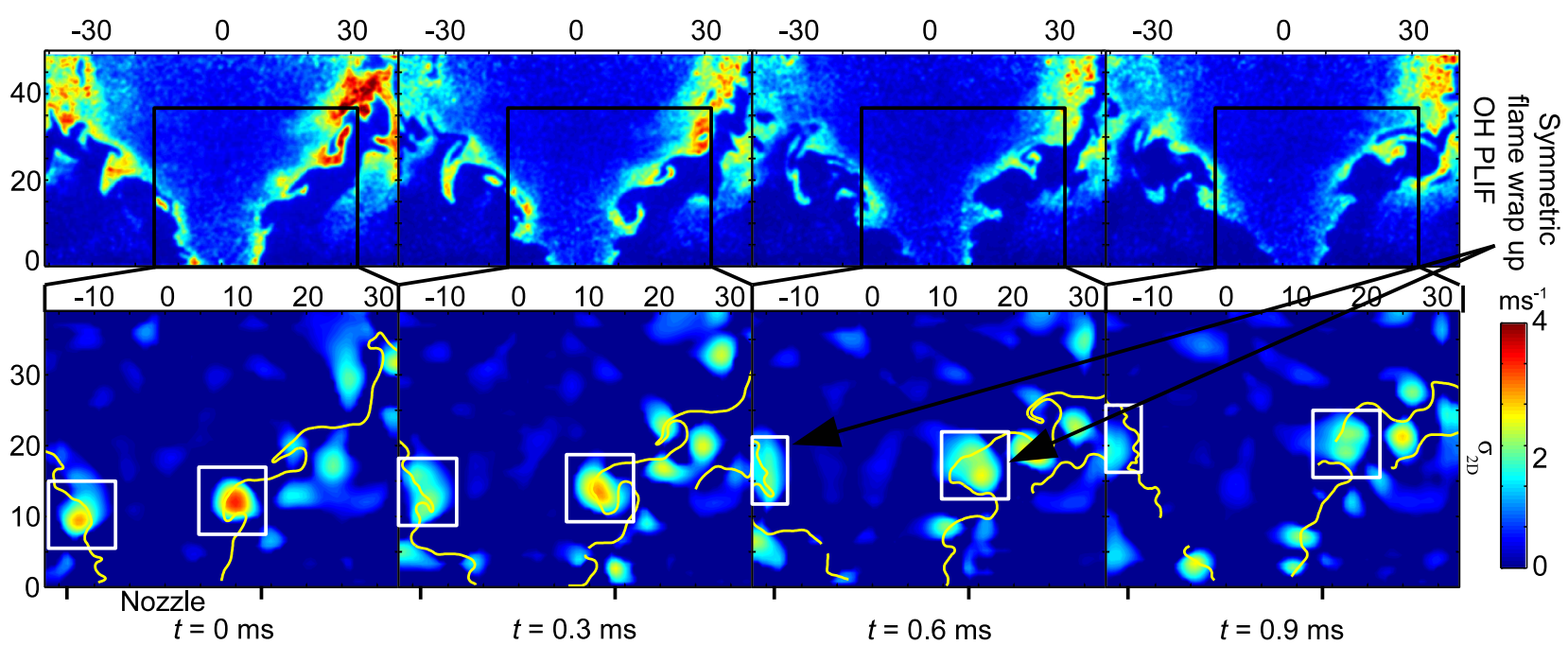

Fig. 4: Typical dynamics of the OH PLIF and swirling strength for Case $1 \mathrm{R}\left(P_{t h}=10 \mathrm{~kW}\right.$, reacting). There is a symmetric vortex shedding that caused flame wrap up. Axis units are millimeters.

caused by a precessional instability of the swirling inflow jet, which caused a precessing azimuthal non-uniformity in the reactant flow rate through the nozzle. Additionally, the HVC caused an asymmetric wrap-up of the flame near the nozzle exit. As time progressed, the position of the HVC and jet asymmetry rotated around the nozzle, the heat release asymmetry shifted, and the HVC interacted with the flame at a different location. It is noted that the heat release asymmetry does not appear to move with the $\mathrm{HVC}$; at times when the $\mathrm{HVC}$ was in mirrored positions across the burner nozzle, the $\mathrm{OH}^{*}$ field was not mirrored. Steinberg et al. [8,9] showed that understanding such asymmetries requires simultaneously considering the phase in the HVC motion and the phase in the thermo-acoustic oscillation, which do not occur at the same frequency.

The low-power non-reacting Case 1NR contained an HVC with a similar structure to that in Fig. 2 (not shown). However, no HVC was present in the corresponding reacting case (Case 1R), as shown in Fig. 4. The OH* fields are not shown since they did not vary in a significant manner over the time sequence. The planar images show a pair of vortices being shed from the shear layers between the inflow and CRZ. This represents a slice through a 3D toroidal structure. The vortex interacts with the flame, causing a roughly symmetric flame corrugation. It will be shown below that this process is periodic at the burner thermo-acoustic frequency.

\subsection{Frequency and spectral power analysis}

To quantify the oscillatory flow dynamics identified above, spatio-temporal proper orthogonal decomposition (ST-POD) analysis was applied. Details of the ST-POD method applied here can be found in Refs. [8, 9]. Briefly, ST-POD analysis from a sequence of $n$ temporally resolved planar measurements of the fluctuation of a variable, $\xi^{\prime}$, provides a set of $n$ spatial eigenmodes $\left(M_{j}(x, y)\right)$, temporal coefficients $\left(a_{j}(t)\right)$, and eigenvalues $\left(\lambda_{j}\right)$ such that the eigenmodes form an orthogonal basis for the original data set. The eigenvalues represent the contribution of the modes to the overall 'energy' $\left(\xi^{\prime} \cdot \xi^{\prime}\right)$ of the original data, and the particular property of POD analysis is that it provides optimal energy convergence. That is, the sum of the highest $k$ eigenvalues for the POD basis is larger than for any other orthogonal basis. Assuming that the eigenvalues are sorted in descending order, the first modes represent the dominant flow features. The temporal history of $\xi$ from the $k$ most dominant modes is given by $\xi_{k}\left(x, y, t_{i}\right)=\bar{\xi}+\sum_{j=1}^{k} \lambda_{j} a_{j}\left(t_{i}\right) M_{j}(x, y)$.

The dynamics of the most energetic flow structures can be quantified using the power spectra of the most energetic ST-POD 


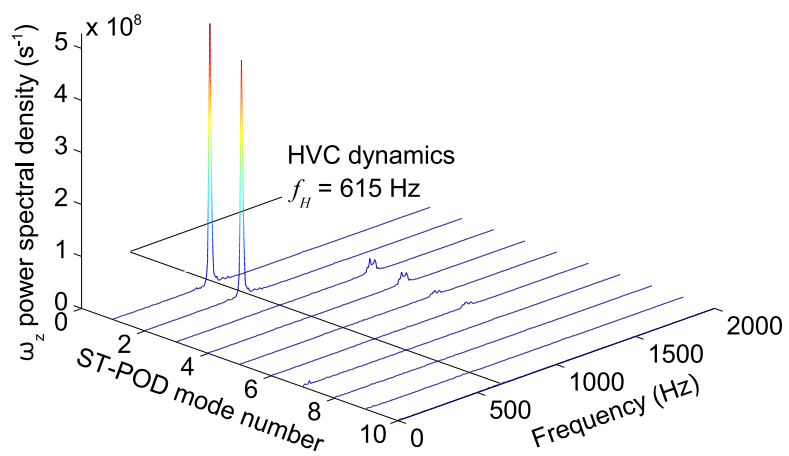

(a) Case 5NR - Non-reacting

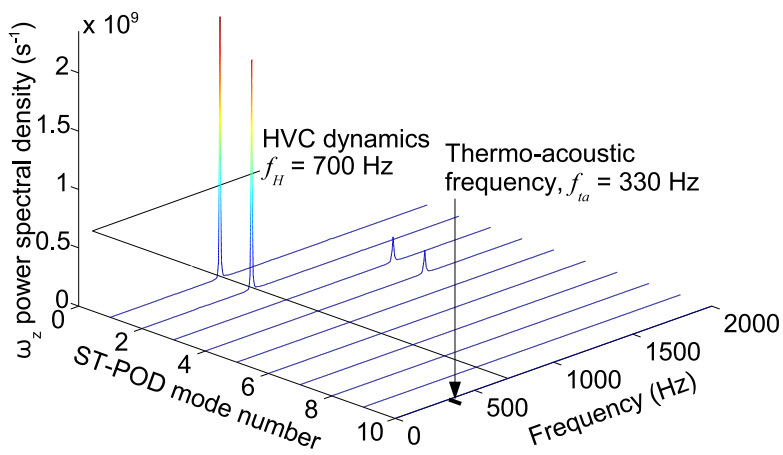

(b) Case 5R - Reacting

Fig. 5: Power spectra of the first ten vorticity ST-POD modes for Case $5\left(P_{t h}=30 \mathrm{~kW}, \phi=0.7\right)$. The frequency of the thermo-acoustic pulsation is indicated for the reacting case.

modes. That is, the instantaneous fluctuation amplitude associated with a given mode, $j$, is $\lambda_{j} a_{j}(t)$. The power spectrum of $\lambda_{j} a_{j}(t)$ provides the power content associated with the flow structure dynamics represented in basis mode $j$. All spectra were calculated using Welch's method [30].

Figure 5 shows the power spectra for the first ten modes from the vorticity ST-POD for Cases $5 \mathrm{NR}$ and $5 \mathrm{R}$. The vorticity spectra are shown (as opposed to $\sigma_{2 D}$ ) because the amplitudes of these spectra have a direct connection to the enstrophy. In general, the vorticity and swirling strength spectra contained identical information. For the reacting case, the frequency of the thermo-acoustic pressure oscillations measured from the microphone probes $\left(f_{t a}\right)$ also is indicated, from which it is clear that the dominant flow dynamics are not directly linked to the thermo-acoustics. Comparing the frequency of the spectral peak to the period of the HVC motion observed in Fig. 3, it appears that these modes represent the HVC. Figure 6 shows the vorticity field reconstructed from the first two ST-POD modes in Case 5R over one oscillation of the mode coefficients, directly demonstrating that these modes represent the motion of the HVC. The spectral peaks of the most energetic (lowest) modes are at the fundamental HVC frequency, while the higher modes are at harmonics. Both the HVC frequency and peak power in the reacting case were higher than in the non-reacting case.

Figure 7 shows the vorticity ST-POD spectra from Cases $1 \mathrm{NR}$ and $1 \mathrm{R}$. In these low thermal power cases, there were considerable differences between the non-reacting and reacting flow structures. An HVC was present in the non-reacting case, and the energetic POD modes represent the HVC. The frequency of the HVC motion is lower than in Case 5NR; it will be shown below that these Cases $1 \mathrm{NR}$ and $5 \mathrm{NR}$ are at a constant Strouhal number.

In the reacting case, no HVC was formed (see Fig. 4). The spectra show that the dominant flow structure dynamics were at the thermo-acoustic frequency and contained considerably less energy than in the non-reacting case. These modes represent 


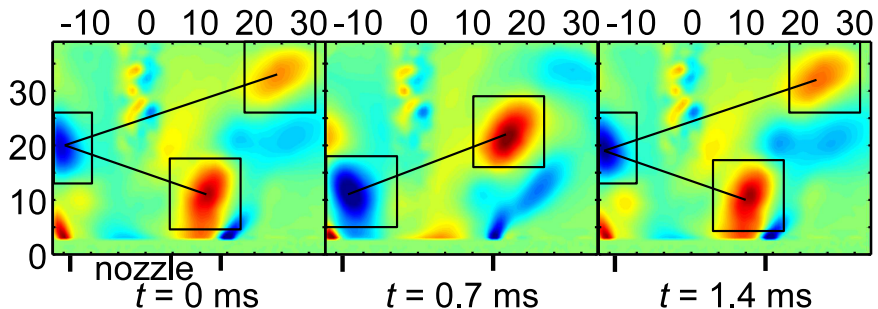

Fig. 6: Vorticity field reconstruction from first two modes of ST-POD analysis in Case 5R. Colormap as in Fig. 4 but scaled between -20000 to $20000 \mathrm{~s}^{-1}$

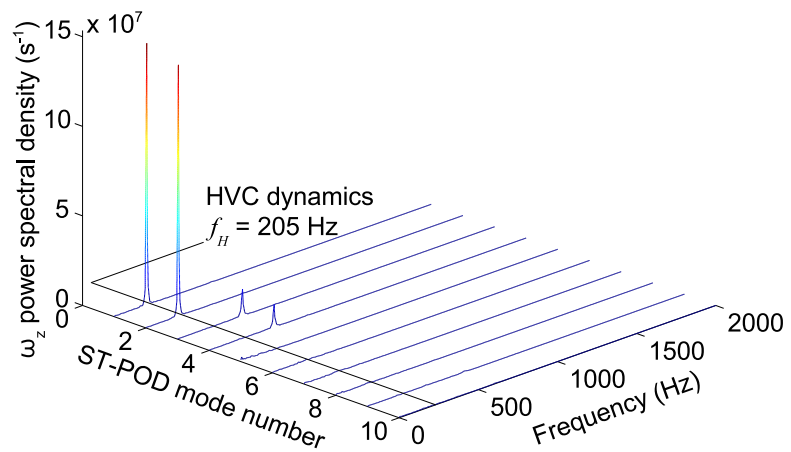

(a) Case $1 \mathrm{NR}$ - Non-reacting

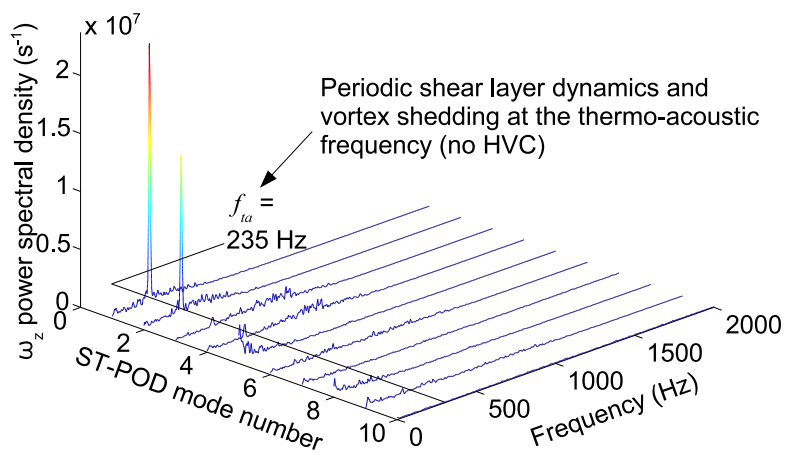

(b) Case 1R - Reacting

Fig. 7: Power spectra of the first ten vorticity ST-POD modes for Case $1\left(P_{t h}=10 \mathrm{~kW}, \phi=0.7\right)$. The frequency of the thermo-acoustic pulsation is indicated for the reacting case.

the shear layer dynamics and periodic vortex shedding at the thermo-acoustic frequency.

Figure 8 shows the frequency from the first vorticity ST-POD mode, plotted against the bulk flow velocity $u_{b}$ (volumetric flow rate/nozzle exit area) for all cases. Also shown are the thermo-acoustic frequencies for the reacting cases. The different markers at similar positions represent different repetitions of the experiment at the same conditions.

In all non-reacting cases, an HVC occurred and its frequency $\left(f_{H, N R}\right)$ was represented by the first ST-POD mode. The HVC frequency increased linearly with the flow rate, indicating a constant HVC Strouhal number of $\mathrm{St}_{H, N R}=f_{H, N R} d_{n} / u_{b}=0.78$, where $d_{n}$ is the nozzle diameter.

In the reacting cases, two different phenomena are represented by the first ST-POD mode frequency. At some conditions, combustion prevented formation of the HVC. In these cases, the first ST-POD mode represented a periodic vortex shedding from the shear layers at the thermo-acoustic frequency; the frequency of the first ST-POD mode was the same as the thermoacoustic frequency. From Fig. 8, it can be seen that no HVC occurred at low thermal powers $\left(P_{t h} \leq 15 \mathrm{~kW}, u_{b}<12 \mathrm{~m} / \mathrm{s}\right)$ and at the highest equivalence ratio (Case 5iii, $\left(P_{t h}=30 \mathrm{~kW}, \phi=0.8, u_{b} \approx 20.2 \mathrm{~m} / \mathrm{s}\right)$ ). An HVC occurred for the remainder 


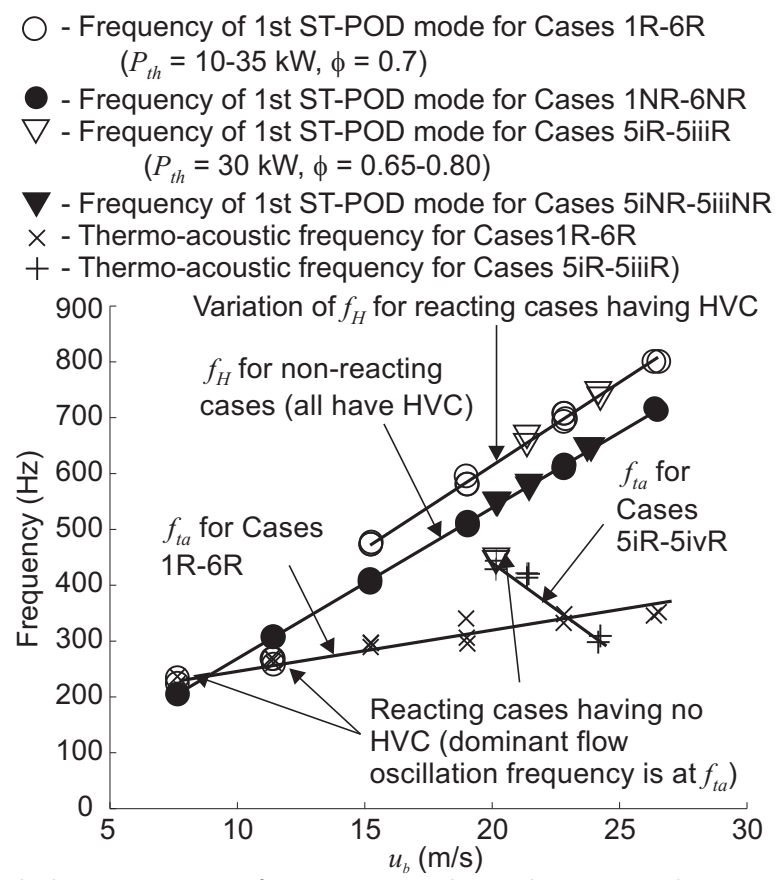

Fig. 8: Variation of flow-structure and thermo-acoustic frequencies with combustor conditions. Multiple symbols at similar locations represent multiple repetitions of the same case.

of the cases and had a frequency higher than the thermo-acoustics. For all such cases, the HVC frequency increased linearly with the flow rate, with a constant Strouhal number of $\mathrm{St}_{H, R}=0.88$ regardless of the thermal power or equivalence ratio.

From the above discussion, it is clear that combustion alters the flow field such that the HVC does not form at certain conditions. However, the strength of the HVC (swirling strength, amplitude of the power spectrum peaks) for every reacting case having an HVC was greater than was found in the corresponding non-reacting case. It therefore appears that combustion may damp the HVC in some cases and excite the HVC in others. Figure 9 shows the vorticity energy associated with the first two ST-POD modes for each case:

$$
\Pi_{12}=\int_{0}^{3900 \mathrm{~Hz}}\left(\pi_{1}(\omega)+\pi_{2}(\omega)\right) d \omega
$$

where $\pi_{j}(\omega)$ is the vorticity power spectrum for ST-POD mode $j . \Pi_{12}$ essentially represents the resolved component of the enstrophy $\left(\omega_{z}^{2}\right)$ contained in modes 1 and 2 . The frequency upper bound in the integral is set by the number of samples used to compute the power spectrum. However, all spectra contained a single peak at less than $1 \mathrm{kHz}$ and there was no significant energy content at higher frequencies. Also shown is the root-mean-squared amplitude of the pressure oscillation for each reacting case. The data are plotted versus thermal power and the equivalence ratio is indicated for the different reacting cases at $P_{t h}=30 \mathrm{~kW}$.

In the non-reacting cases (which all had an $\mathrm{HVC}$ ), $\Pi_{12}$ increased monotonically with the flow velocity. In the reacting cases, the behavior of $\Pi_{12}$ depended on whether an HVC was present. For cases having an HVC, $\Pi_{12}$ was significantly greater than for the corresponding non-reacting case. However, $\Pi_{12}$ in cases where the HVC was not present in the reacting flow was considerably lower than the corresponding non-reacting cases (which had an HVC). This attribute is most apparent for Case 5iii $(30 \mathrm{~kW}, \phi=0.8)$, but also is present for Cases 1 and $2(10 \mathrm{~kW}$ and $15 \mathrm{~kW}, \phi=0.7)$. For reacting cases with an HVC, $\Pi_{12}$ increased with the thermal power. However, within the $30 \mathrm{~kW}$ cases, the greatest $\Pi_{12}$ was at $\phi=0.75$, which did not have the highest flow rate. 


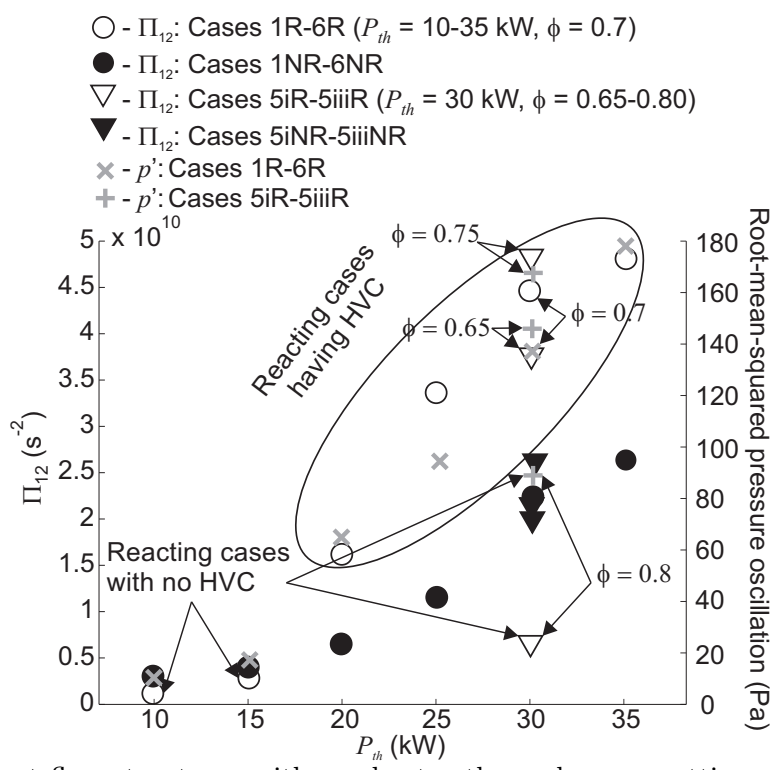

Fig. 9: Variation of energy of dominant flow structures with combustor thermal power setting. Symbols are the average over multiple runs at the same conditions.

Also plotted in Fig. 9 is the root-mean-squared amplitude of the pressure fluctuation for each reacting case. These data clearly show a direct correspondence between the presence of the HVC and the acoustic amplitude. Cases with HVCs had considerably stronger pressure oscillations than cases with toroidal vortex shedding. Case 5 iii $(30 \mathrm{~kW}, \phi=0.8)$ did not have an HVC, had a much lower $\Pi_{12}$, and weaker pressure oscillations than the other $30 \mathrm{~kW}$ cases. This clearly shows that there is a direct relationship between the presence of the HVC and the thermo-acoustic state of the combustor. It should be noted that this plot does not directly imply that the pressure oscillation amplitude scales with the enstrophy of the HVC. Many other combustor properties that influence the thermo-acoustic instability also change with the thermal power. Future analysis of these flames will focus on mapping the thermo-acoustic energy transfer caused by flow-flame interactions in order to elucidate these relationships.

\section{Conclusions}

The geometry and dynamics of the dominant flow structures in a perfectly premixed swirl-stabilized gas turbine model combustor were investigated using multi-kHz laser diagnostics over a range of thermal powers and equivalence ratios. The flow fields were found to contain either periodically shed toroidal vortices or helical precessing vortex cores, depending on the flow conditions and whether the flow was reacting or non-reacting. In all non-reacting cases, the most energetic dynamic flow structure was a helical vortex core that rotated around the combustor at a fixed Strouhal number. However, igniting the burner had a major effect on the flow. In the majority of cases, the helical vortex remained, but increased substantially in frequency and strength (as described by a proper orthogonal decomposition analysis). The Strouhal number of this vortex was constant for all reacting cases, regardless of the thermal power or equivalence ratio, and higher than in the non-reacting cases. However, in some reacting cases, combustion prevented formation of the helical vortex core. This was true for the two lowest thermal power cases and the highest equivalence ratio case. At these conditions, the dominant flow structure was a toroidal vortex that was shed from the nozzle shear layer at the thermo-acoustic frequency.

A relationship was found between the amplitude of the pressure oscillations and the geometry/strength of the dominant flow structures. There was a distinct jump in pressure oscillation amplitude between the $15 \mathrm{~kW}$ cases with the toroidal vortex 
and the $20 \mathrm{~kW}$ case with the helical vortex. Moreover, the $30 \mathrm{~kW}$ cases with helical vortices had substantially higher pressure oscillation amplitudes than the $30 \mathrm{~kW}$ case with the toroidal vortex. Hence, the helical vortex cores in this burner generally contribute to exciting thermo-acoustic oscillations. Future work will investigate the thermo-acoustic energy transfer caused by these interactions in order to quantify this effect.

\section{Acknowledgement}

The financial support from the German Research Foundation within the CRC606 is gratefully acknowledged.

\section{References}

[1] S. M. Correa, Proc. Combust. Inst. 27 (1998) 1793-1807.

[2] A. Lefebvre, Gas Turbine Combustion, 2nd Edition, Taylor and Francis, 1999.

[3] T. Lieuwen, V. Yang (Eds.), Combustion instabilities in gas turbine engines: Operational experience, fundamental mechanisms, and modeling, AIAA, 2006.

[4] T. Poinsot, A. C. Trouve, D. P. Veynante, S. M. Candel, E. J. Esposito, J. Fluid Mech. 177 (1987) 265-292.

[5] K. Schadow, E. Gutmark, Prog. Energy Combust. Sci. 18 (1992) 117-132.

[6] A. Ghoniem, S. Park, A. Wachsman, A. Annaswamy, D. Wee, H. Altay, Proc. Combust. Inst. 30 (2005) $1783-1790$.

[7] J. O'Connor, T. Lieuwen, Combust. Sci. Technol. 183 (2011) 427-443.

[8] A. M. Steinberg, I. Boxx, M. Stöhr, C. D. Carter, W. Meier, Combust. Flame 157 (2010) 2250-2266.

[9] A. M. Steinberg, I. Boxx, M. Stöhr, W. Meier, C. D. Carter, AIAA J. 50 (4) (2012) 952-967.

[10] R. Weber, J. Dugué, Prog. Energy Combust. Sci. 18 (1992) 349-367.

[11] C. O. Paschereit, E. Gutmark, W. Weisenstein, Phys. Fluids 11 (1999) 2667-2678.

[12] O. Lucca-Negro, T. O’Doherty, Prog. Energy Combust. Sci. 27 (2001) 431-481.

[13] S. Roux, G. Lartigue, T. Poinsot, U. Meier, C. Bérat, Combust. Flame 141 (2005) 40-54.

[14] C. E. Cala, E. C. Fernandes, M. V. Heitor, S. I. Shtork, Exp. Fluids 40 (2006) 267-276.

[15] S. Wang, V. Yang, G. Hsiao, S.-Y. Hsieh, H. C. Mongia, J. Fluid Mech. 583 (2007) 99-122.

[16] N. Syred, Prog. Energy Combust. Sci. 32 (2006) 93161.

[17] Y. Huang, V. Yang, Prog. Energy Combust. Sci. 35 (2009) 293-365.

[18] A. Lacarelle, T. Faustmann, D. Greenblatt, C. O. Paschereit, O. Lehmann, D. M. Luchtenburg, B. R. Noack, J. Eng. Gas Turb. Power 131 (2009) 031504.

[19] P. Iudiciani, C. Duwig, Flow Turb. Combust. 86 (2011) 639-666.

[20] W. Meier, P. Weigand, X. Duan, , R. Giezendanner-Thoben, Combust. Flame 150 (2007) 2-23.

[21] P. Weigand, W. Meier, X. R. Duan, M. Aigner, J. Eng. Gas Turb. Power 129 (2007) 664-671.

[22] W. Meier, I. Boxx, M. Stöhr, C. D. Carter, Exp. Fluids 49 (2010) 865-882.

[23] C. M. Arndt, A. M. Steinberg, I. Boxx, W. Meier, M. Aigner, Proceedings of ASME Turbo Expo Glasgow, UK (2010) GT2010-22830.

[24] G. Lartigue, U. Meier, C. Bérat, Appl. Therm. Eng. 24 (2004) 1583-1592.

[25] J. Galpin, A. Naudin, L. Vervisch, C. Angelberger, O. Colin, P. Domingo, Combust. Flame 155 (2008) $247-266$.

[26] B. Fiorina, R. Vicquelin, P. Auzillon, N. Darabiha, O. Gicquel, D. Veynante, Combust. Flame 157 (2010) $465-475$.

[27] B. Franzelli, E. Riber, L. Y. M. Gicquel, T. Poinsot, Combust. Flame 159 (2012) 621-637. 
[28] N. Docquier, S. Candel, Prog. Energy Combust. Sci. 28 (2002) 107-150.

[29] J. Zhou, R. J. Adrian, S. Balachandar, T. M. Kendall, J. Fluid Mech. 387 (1999) 353-396.

[30] P. Welch, IEEE Trans. Audio Electroacoust. AU-15 (1967) 70-73. 